\title{
Comparative analysis of techniques for diagnostics of phase singularities
}

\author{
Felde Ch.V., Polyanski P.V. and Bogatyryova H.V. \\ Department of Correlation Optics, Chernivtsi National University, \\ 58012 Chernivtsi, Ukraine, polyanskii@itf.cv.ua
}

Received: 23.11.2007

\begin{abstract}
A comparative analysis of several techniques used for diagnostics of phase singularities in the optical vortex beams and fields is performed. Both the advantages and shortcomings of implementation and application of those techniques are discussed.
\end{abstract}

Keywords: singular optics, wavefront dislocations, interference, diffraction, Young's interference experiment

PACS: $42.15 . \mathrm{Dp}$, 78.20.Fm, $65.57 \mathrm{Fg}$

UDC: 535.31

\section{Introduction}

One of the urgent problems of singular optics consists in development of special techniques for detection and diagnostics (i.e., determination of the magnitude and the sign of topological charge) of phase singularities in vortex-supporting beams and optical fields. Sometimes conventional interference techniques $[1,2]$ are not efficient enough or even inapplicable. The examples are studies of partially spatially coherent combined singular beams, which are inhomogeneous in polarization, or polychromatic beams. Indeed, application of interference techniques is impossible when diagnosing partially spatially coherent singular beams resulting from mixing of mutually incoherent Laguerre-Gaussian modes. This is because it would be impossible to form a reference wave coherent with all the constituting modes simultaneously. Application of the classical interference techniques for diagnostics of phase singularities in polychromatic fields becomes extraordinary complicated since a precise adjustment of optical arrangement is needed.

In this work we consider a number of techniques for detecting and diagnosing of phase singularities, which are both novel and conventional. We analyse their advantages and shortcomings that concern the implementation and practical applications. Moreover, we examine a self-descriptiveness and unambiguity of the results obtained with those techniques. 


\section{Analysis}

\subsection{Interference technique}

A conventional technique for diagnostics of phase singularities in the optical fields is an interference one [1,3]. It presumes employing a reference beam mutually coherent with the singular beam and observation of on-axis or off-axis interference patterns. When applying the interference technique to the analysis of "elementary", deterministic optical vortices of the kind of Laguerre-Gaussian modes, one enforces interference of the beam bearing one or several phase singularities with a plane or spherical reference beam. In practice, it is convenient to use a spread Gaussian beam for this purpose. Both the offaxis and on-axis schemes are widely utilized. The fringes formed in such a manner can be straight, with typical "bifurcations", or spiral. This depends on the inclination and curvature of wavefront of the reference beam [3], as shown in Fig. 1 and 2. Let us note that the number of dark fringes originating from the centre (for the off-axis interference scheme -

Fig. 1) is equal to the module of topological charge, $|m|$, whereas the sign of the vortex (i.e., the sign of the topological charge) is determined by the direction of bifurcation of interference "forklets" (up or down), taking into account the propagation direction of the reference beam with respect to the interferogram axis.

If one implements the on-axis technique (see Fig. 2) for diagnostics of phase singularities, then the module and sign of the topological charge of the vortex beam are determined from the number and direction of twirling of the "blades" with respect to the interferogram axis. In this case one must account for the relation between the curvature radii of the reference beam and the beam under test [1, 3, 4]. It is clear that in the both cases interference test of the vortex beams and fields assumes a complicated and precisely adjusted optical apparatus. So, according to the experimental estimations [4], the on-axis interference pattern is observed if the angle between the axes of the reference and singular beams does not exceed $5 \cdot 10^{-4} \mathrm{rad}$ (i.e., about $1.5^{\prime \prime}$ ) and the ratio of the curvature radii for those beams exceeds unity, $R_{S} / R_{r e f}>1$.

Thus, the interference technique for the diagnostics of phase singularities provides
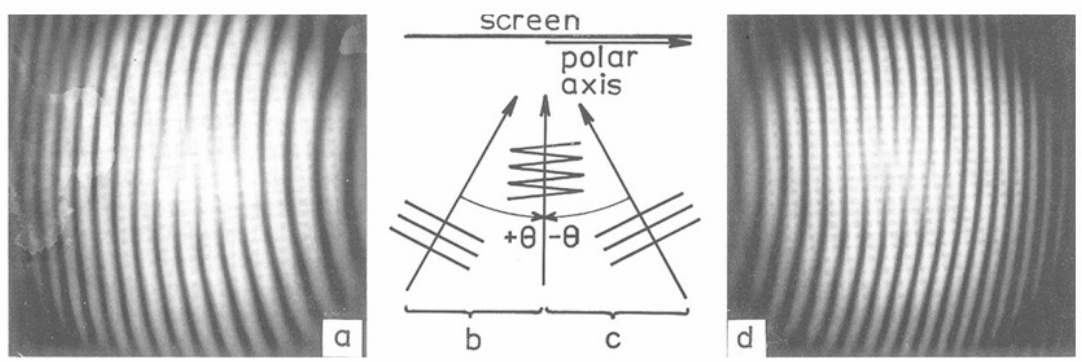

Fig. 1. Off-axis interferograms $(a, d)$ of the wavefront with a unit-charged screw dislocation $(m=1)$ and the corresponding interference schemes $(b, c)$.

Ukr. J. Phys. Opt. 2008, V9, №2 


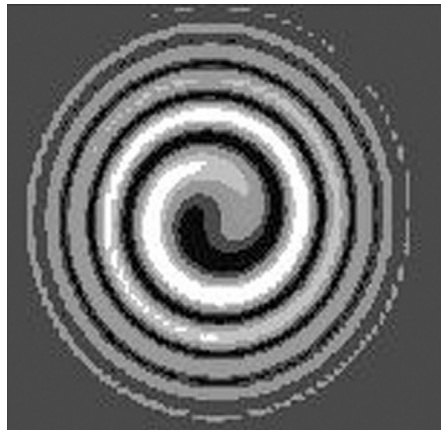

Fig. 2. On-axis interferogram for a single-charged optical vortex. information on both the module and the sign of the topological charge of vortex beams. However, unambiguous results are obtained only if one has a priori data on the arrangement and parameters of the test beam and the reference beam. Besides, interpretation of the results obtained using the offaxis technique (e.g., the sign of the topological charge of the vortex) depends upon orientation of the corresponding pattern. For instance, rotation of the pattern shown in Fig. 1a or 1d by $180 \mathrm{deg}$ would result in the vortex of opposite sign.

\subsection{Dove-prism technique}

This approach consists in interference of Laguerre-Gaussian mode with its mirror replica [5]. It is known [2] that the mirror reflection results in reversing sign of the topological charge. Therefore, when a vortex beam is divided into two partial beams and one additional reflection is provided for one of them, then the two partial beams would exhibit the opposite signs of the topological charge. The Dove-prism technique is based on summation and interference comparison of those modes. The idea of the technique is to compare the test beam with its mirrored replica ("auto-reference technique"). This means that the technique does not involve additional reference beams. As a consequence, the two partial beams are peer, and the information concerned with the sign of the topological charge of the initial vortex is lost.

The relevant experimental arrangement is shown in Fig. 3. A Dove prism inserted into one arm of Mach-Zehnder interferometer inverts the input Laguerre-Gaussian beam, which interferes coaxially with the initial (doubly mirrored) beam at the interferometer output. This gives rise to interference pattern in the shape of fringes, with $2 m+1$ forklets in the centre. Notice that this technique allows deriving only the module of the topological charge of the singularity under test, provided that the number of interference fringes originating from the centre of interference pattern is known. It is therefore impos-

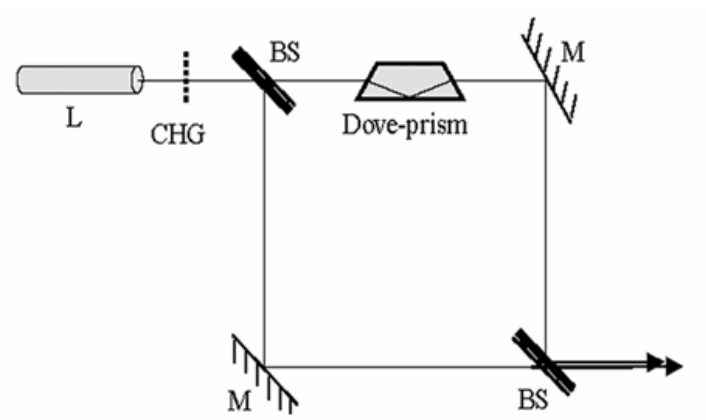

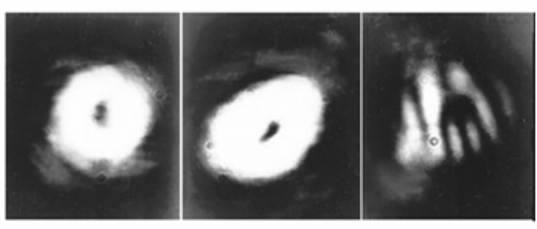

(a) (c)

Fig. 3. Interference scheme including the Dove prism for coherent superposition of two oppositely charged vortices ( $a$ and $b$ ) and the result of their superposition (c). 
sible to use this technique for determination of sign of the mode charge. Furthermore, the technique is applicable to diagnostics of the isolated vortices alone but not for studying the phase singularities of speckle fields and partially coherent singular beams.

\subsection{Diffraction technique}

Another technique aimed at diagnostics of the optical vortices has been suggested in Ref. 6. It is based upon the "strip" Young's interference experiment $[6,7]$. The idea of experimental investigation of the vortex optical beams can be elucidated using the notations of Fig. 4. An opaque strip is placed on the path of the beam under study, symmetrically to its centre, and the interference fringes arising in geometrical shadow of the strip are examined. Following the Young-Rubinowicz model of the diffraction phenomena, we consider these interference fringes as a result of superposition of the edge diffraction waves, which are thought as being re-transmitted by the strip edges. Taking the stationary phase principle into account, we regard the fringes at any height $r$ as being produced by the edge re-transmitters localized at this height alone.

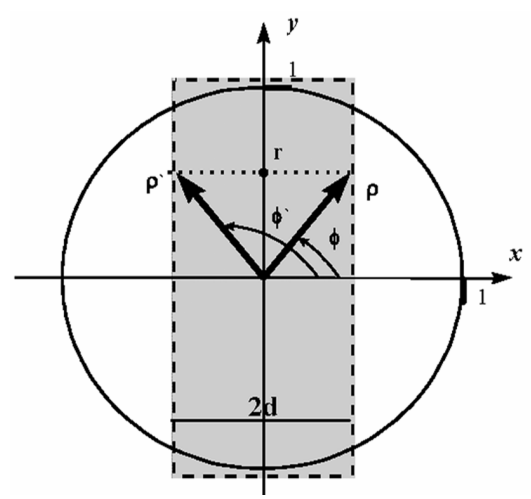

Fig. 4. Notations used for analysing the Young's strip interference experiment.
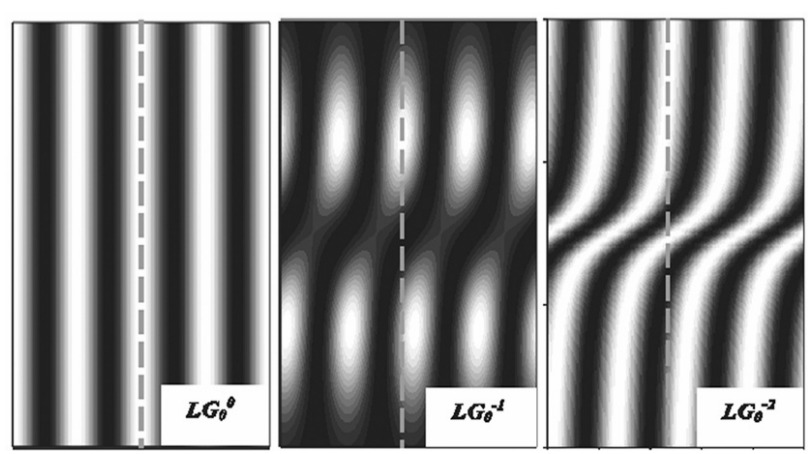

Fig. 5. Simulation of the Young's interference fringes for the vortexless, singly-charged $(m=-1)$ and the doubly-charged $(m=-2)$ Laguerre-Gaussian modes.

In Fig. 5 one can see the interference pattern observed behind the diffraction strip positioned in front of both vortex-free and vortex-supporting beams. In the first case (vortex-free beam - see Fig. 5a) the phases of the edge re-transmitters on the right and left sides are the same. So, one can expect that straight Young's interference fringes are observed within the geometrical shadow, with a maximum along the mean line of the shadow. They are produced by superposition of the right- and left-sided wavelets. If the test beam supports the $m$-charged vortex, one should observe bent interference fringes, with a maximum (if $m$ is even) or zero (if $m$ is odd) at the equator (see Fig. $5 \mathrm{~b}$ and $5 \mathrm{c}$ ). In general, the phase of the Young's interference fringes for the case under consideration obeys the following rule: 


$$
\Delta \varphi(r, d)=m[\pi \pm \arctan (r / d)],
$$

where $d$ is the halfwidth of the diffraction strip.

The basic experimental setup is shown in Fig. 6. A vortex-free mode $L G_{0}^{0}$ of $\mathrm{He}-\mathrm{Ne}$ laser illuminates a computer-generated hologram, which is computed in order to reconstruct single-charged optical vortices of opposite signs at the plus-minus first diffraction orders. In the region where the diffraction spectra are separated, we introduce an opaque

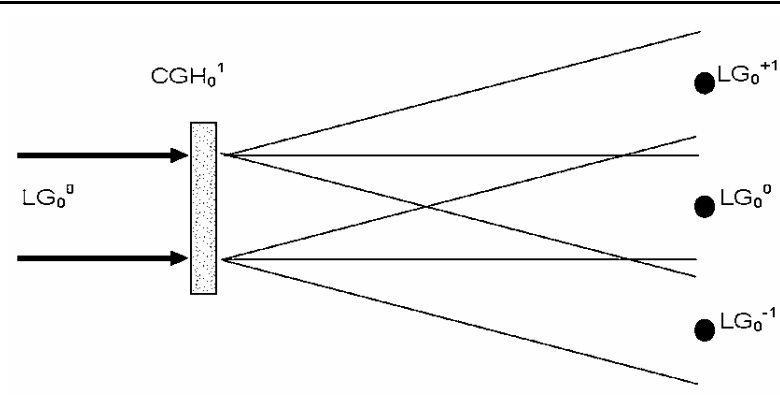

Fig. 6. Experimental setup employed for diffraction diagnostics of the vortex-bearing optical beams.

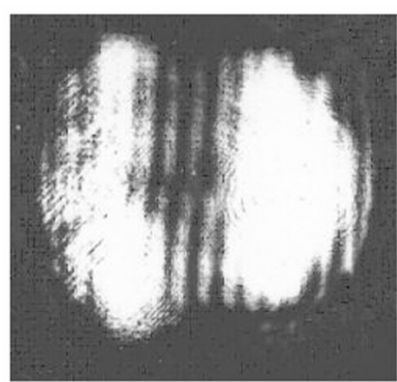

(a)

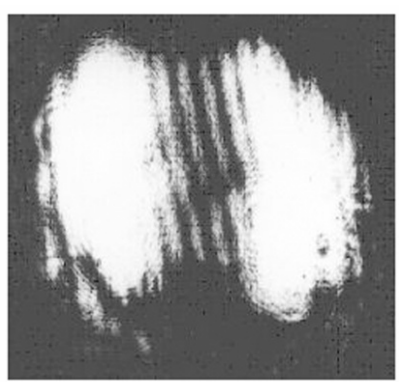

(b) screen in front of each of the diffraction orders. Furthermore, we use a metallic needle as a diffraction screen. Diffraction testing of the $L G_{0}^{-1}$ mode supporting counter-clockwise vortex and the $L G_{0}^{+1}$ mode supporting clockwise vortex are illustrated in Fig. $7 \mathrm{a}$ and $7 \mathrm{~b}$, respectively.

Fig. 7. Diffraction tests of oppositely charged vortex beams: $L G_{0}^{-1}$ mode (a) and $L G_{0}^{+1}$ mode (b).

As a conclusion, the diffraction technique for diagnostics of phase singularities provides comprehensive information on the beam singularity, viz. the information on both the module parameters of the topological charge of the observed vortex and its sign. The mentioned quantities are derived respectively from the magnitude and the direction of the Young's interference fringes in the geometrical shadow appearing behind the opaque strip. The technique requires neither additional reference beam nor complicated optical setup. The former fact makes the method convenient when diagnosing vortices in partially spatially coherent [7-9] and polychromatic [10] beams, for which formation of coherent reference waves is problematic or, sometimes, impossible. Contrary to the interference cross-correlation techniques where one compares the phases of singular and reference beams, the diffraction technique is in fact autocorrelation one. Namely, it involves comparison of the phases at different points of one singular beam. That is why 
there is no need in any a priori information on the beam under study. In contrast to the interference technique, the results obtained with the diffraction technique (see Fig. 7) are unbiassed in a sense that they are invariant with respect to the orientation of the pattern. Really, the direction of bending of the Young's interference fringes is always the same, thus enabling unambiguous determination of the sign of topological charge of the beam under test.

\subsection{Wave-guide technique}

One more technique has been suggested recently for determining the structure of the optical vortices. It originates from the model of planar waveguide with a leaky mode [11]. Similarly to the Dove-prism and the diffraction techniques, here the reference beam is not used, although the principle of detection of the vortex is different. Multi-beam interference occurring in a planar waveguide results in high angular resolution, so that such an "interferometer" can be used for diagnostics of phase singularities.

A planar waveguide with a leaky mode is a set of two equal-sided glass prisms, with an immersion liquid between them. The refraction index of the immersion is less than that of the prisms. The surfaces of the prisms sandwiching the immersion in between are parallel. As a result, a narrow dark fringe appears in the cross-section of reflected beam, which is observed both in the near and far fields. This provides a possibility for observing a line of zero intensity across the beam that represents a non-localized (infinitely extended) wave dislocation. Scanning the optical beam by such a fringe of zero intensity, one can determine the wave front structure of the beam under test, following from the fringe shape.

As seen from Fig. 8, the parameters (the module and sign) of the vortex beam in the presence of singularity are determined from typical bending of the fringe of zero intensity. Similarly to the diffraction technique, the pattern is invariant with respect to rotation by $180 \mathrm{deg}$. Therefore the direction of bending of the zero-intensity fringe is informative and the technique requires no a priori information.

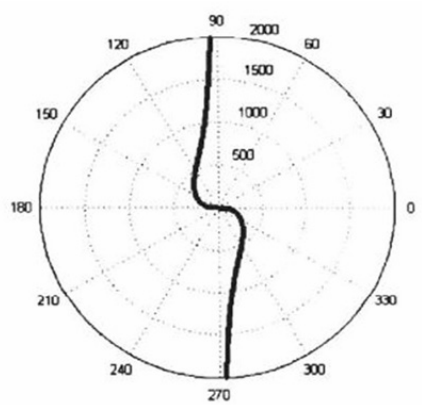

(a)

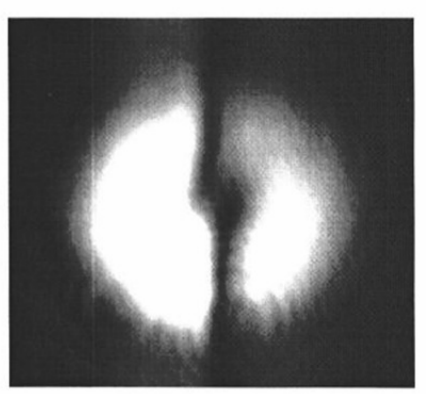

(b)
Fig. 8. Modulation of dark line in the geometrical optics approximation: the beam waist is before the waveguide (a) and the beam image reflected from the waveguide (b).

Thus, using the waveguide technique for diagnostics of phase singularities in the optical beams, one can determine the parameters of vortex under study. The technique has recently been implemented for the white-light vortices [12]. However, practical realiza- 
tion of this approach is rather sophisticated. In order to provide multi-beam interference in a thin layer of immersion liquid (e.g., glycerin), one must use precise goniometric equipment. Besides, the test beam must have a diffraction-limited divergence. Moreover, one must take into account that a unique high resolution $\left(6 \times 10^{-5}\right.$ for $\mathrm{He}-\mathrm{Ne}$ laser $)$ is predicted by the geometrical theory of the technique alone (for the case of infinitely narrow dark line). At the same time, within the wave optics approximation and even more so in the experiment, such the resolution cannot be achieved, whereas the infinitely narrow "black" line is surrounded by relatively broad area of non-zero, though very low, intensity (see Fig. 8b).

\subsection{Chromascope technique}

A quite novel technique for processing of phase singularities in the polychromatic speckle fields arises from a concept of chromascope [13, 14]. The latter is intended for explanation of a universal colour gamut seen by a human observer near an isolated zero, viz., within the area where the complex amplitude $\psi(R, k)$ varies linearly with the position $R$ (over the spatial range considered) and the wavenumber $k$ (over the visible range). In is remarkable that in this case "Approximately circular regions of colours, including intense blue, red and yellow, separated by a large white circle, merge into an unsaturated "asymptotic white"... the region in the total gamut of possible colours that the universal pattern occupies is rather small; most notably, there is no green" (see Fig. 9). The concept of

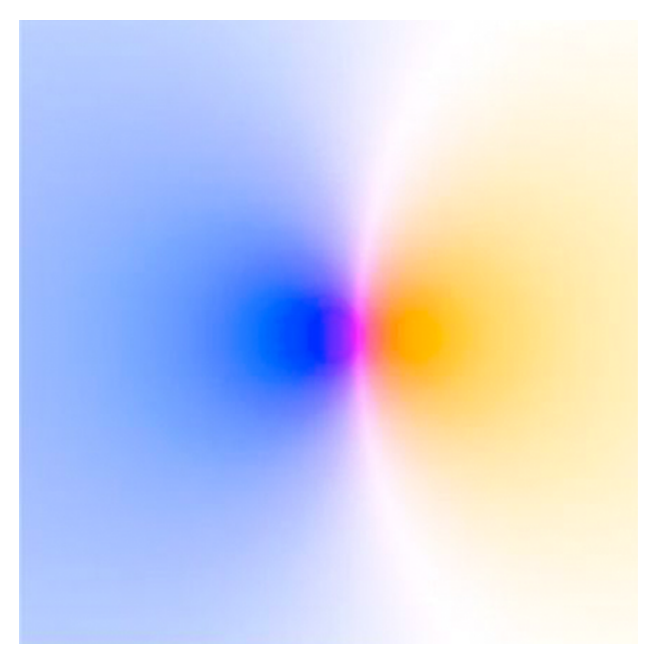

Fig. 9. Universal colour pattern seen near the isolated phase singularity. chromascope is implemented using the following equation:

$$
\left(\begin{array}{l}
R \\
G \\
B
\end{array}\right)_{C R}=\left(\begin{array}{l}
R \\
G \\
B
\end{array}\right) / \max (R, G, B),
$$

where we normalize the colours at each point $(\xi, \eta)$ of the field, basing on the maximum intensity for any colour in the RGB scale. This increases the brightness for the points of low intensity, viz., in the vicinity of the amplitude zeroes. Therefore, if all of the three spectral components are of low intensity, then these areas processed by a chromascope appear "white" [15].

The technique considered above can be applied for detecting the vortices in the polychromatic fields from the universal gamut of colours. Nonetheless, it is inapplicable for determining the vortex parameters. Besides, we deal in fact with a software technique assigned just for the polychromatic fields, rather than a universal experimental technique. 


\section{Conclusions}

In this work we have analysed a number of techniques for detection and diagnostics of phase singularities in optical beams and fields. We have argued the advantages and disadvantages of these diagnostic techniques. It has been shown that some of the techniques (in particular, the Dove-prism and the chromascopic ones) are applicable for detecting the phase singularity alone, though they do not provide information on the parameters of that singularity.

The other kind of the techniques (i.e., the interference one) requires some a priori information on the curvature radius of the reference beam (the case of the on-axis technique) or on the mutual orientation of the reference and singular beams (the off-axis technique). It is needed for determining correctly the sign of the topological charge of vortices. Having this information, one can obtain unambiguous and comprehensive results of diagnostics. Such the techniques are the most popular among experimentalists elaborating completely spatially coherent monochromatic fields.

At the same time, the interference techniques become less convenient or even impossible for the domain of partially spatially coherent and polychromatic fields. Some techniques (e.g., the waveguide one) require complicated and high-precision equipment, as well as fine adjustment of the optical apparatus. Practical resolution provided in frame of the technique mentioned above might be considerably less than that predicted by the geometrical optics. Nevertheless, this technique is unbiassed and enables unambiguous diagnostics of optical vortices without any a priori information on the beam parameters. Moreover, it can be applied to white-light vortices, too.

In our opinion, the most flexible technique for the diagnostics of phase singularities is the diffraction one. It is based on the Young's model of the diffraction phenomena (the model of the edge diffraction wave) and the Young's interference experiment in its initial form, with the opaque strip. This technique requires neither a priori information on the tested beam nor complicated optical equipment and. At the same time, it gives unambiguous and comprehensive data on the parameters of singular beam, including the module and the sign of its topological charge. This technique has been successfully used in experimental studies of phase singularities of completely coherent, partially spatially coherent, inhomogeneously polarized and polychromatic light beams.

\section{References}

1. Baranova N B, Zeldovich B Ya, Mamayev A V, Pilipetsky N F and Shkunov V V, 1981. Dislocation of the wavefront of a speckle-inhomogeneous field (theory and experiment). JETP. 33: 1789-1797.

2. Soskin M S and Vasnetsov M V, Singular Optics, in Progress in Optics 42 (E. Wolf, Ed.): 219-276 (2001).

3. Basistiy I V, Soskin M S and Vasnetsov M V, 1995. Optical wavefront dislocations and their properties. Opt. Comm. 119: 604-612.

4. Bogatyryova G V and Soskin M S, 2003. Detection and metrology of optical vortex

Ukr. J. Phys. Opt. 2008, V9, №2 
helical wave fronts. Semicond. Phys., Quant. Electron \& Optoelectr. 6 (2): 254-258.

5. Allen L, Padgett $\mathrm{M} \mathrm{J}$ and Babiker $\mathrm{M}$, The orbital angular momentum of light, in Progress in Optics 40 (E. Wolf, Ed.): 291-371 (1999).

6. Bogatyryova G V, Felde Ch V and Polyanskii P V, 2003. Referenceless testing of vortex optical beams. Opt. Appl. 33: 695-708.

7. Bogatyryova G V, Felde Ch V, Polyanskii P V, Ponomarenko S A, Soskin M S and Wolf E, 2003. Partially coherent vortex beams with a separable phase. Opt. Lett. 28: 878-880.

8. Schouten H F, Gbur G and Wolf E, 2003. Phase singularities of the coherence function in Young's interference pattern. Opt. Lett. 28 (12): 968-970.

9. Gbur G and Visser T D, 2003. Coherence vortices in partially coherent beams. Opt. Commun. 222: 117-125.

10. Polyanskii P V, Optical correlation diagnostics of phase singularities in polychromatic fields, Chapter 2 in Optical Correlation Applications and Techniques (O. Angelsky, Ed.), SPIE Press A168, Bellingham (2007).

11. Marienko I G, Pas'ko V A, Slyusar V V, Soskin M S and Vasnetsov M V, 2002. Investigation of an optical vortex beam with a leaky planar waveguide. Opt. Commun. 213: 1-11.

12. Soskin M S, Polyanskii P V and Arkhelyuk O O, 2004. Computer-synthesized hologram-based rainbow optical vortices. New J. Phys. 6: 196.1-196.8.

13. Angelsky O V, Maksimyak A P, Maksimyak P P and Hanson S G, Interference diagnostics of white-light vortices, Opt. Express, 13: 8179-8183 (2005).

14. Berry M V, Exploring the colours of dark light, New J. Phys., 4: 74.1-74.14 (2002).

15. Leach $\mathrm{J}$ and Padgett $\mathrm{M} \mathrm{J}, 2003$. Observation of chromatic effects near a white light vortex. New J. Phys. 5 (154): 1-7. 